\title{
Necrotizing Fasciitis of the Breast: A Case Report and Literature Review
}

\author{
Neesa Fadaee ${ }^{\mathrm{a}, \mathrm{b}}$, Michelle Ong ${ }^{\mathrm{a}}$, Mohammed Al-Askari ${ }^{\mathrm{a}}$
}

\begin{abstract}
A 51-year-old woman presented to our facility with an open wound in the left breast. This was associated with a hard, non-mobile, tender lesion palpable underneath. The wound contained central necrosis with surrounding purulent discharge and accompanying erythema. Following radical debridement of the breast down to the pectoralis fascia the patient had a vacuum-assisted closure (VAC) device dressing applied. Histological examination was consistent with necrotizing fasciitis of the breast.
\end{abstract}

Keywords: Necrotizing fasciitis; Breast; Infection

\section{Introduction}

Necrotizing fasciitis is a rare, rapidly progressing, potentially fatal infection. It spreads rapidly across fascial planes. Timely diagnosis is essential to decrease morbidity and mortality. Necrotizing fasciitis of the breast is a rare condition with only 18 previously published case reports describing this pathology.

\section{Case Report}

A 51-year-old woman presented to her general practitioner with a 2-week history of a painful left breast lump. She denied any history of trauma. Her past medical history included ischemic heart disease, myocardial infarction, type 2 diabetes, dyslipidemia, hypertension, anxiety, depression, and asthma. Examination demonstrated a firm $30-\mathrm{mm}$ breast lesion at 10 o'clock position in her left breast, $7 \mathrm{~cm}$ from the nipple. No axillary lymphadenopathy was appreciated. She was subsequently referred to a general surgical outpatient clinic and underwent further investigations.

Mammography revealed a poorly circumscribed mass,

Manuscript submitted August 30, 2019, accepted September 16, 2019

${ }^{a}$ General Surgical Department, Gladstone Hospital, Gladstone, QLD, Australia bCorresponding Author: Neesa Fadaee, General Surgical Department, Gladstone Hospital, Gladstone, QLD 4680, Australia.

Email: neesafadaee@gmail.com

doi: https://doi.org/10.14740/jmc3361 measuring $40 \times 24 \mathrm{~mm}$, in the lateral aspect of the left breast at the level of the nipple. There were irregular and stranded contours without suspicious calcifications (Fig. 1). The right breast appeared normal. Ultrasound (US) revealed a lobulated, heterogeneous, hypoechoic mass lesion at the region of palpable abnormality (Fig. 2). The mass measured $31 \times 17 \times 21 \mathrm{~mm}$ with accompanying distortion of the surrounding structures in combination with edema and skin thickening. Given the nature of these imaging findings, the lesion was considered suspicious for malignancy.

Five days following mammogram and US the patient represented to her general practitioner with increasing left breast erythema and swelling. Pus had also begun to be discharged from the upper inner quadrant of the left breast. She was commenced on oral clindamycin for cellulitis and was referred for a US-guided biopsy. US revealed that the lesion had significantly increased in size with marked skin thickening and erythema. This was thought to represent an inflammatory lesion; however concerns remained that this may have occurred secondary to an underlying malignancy. Aspiration of the main lesion was attempted under US guidance with a 19-G needle; however, the highly viscous nature of the pus precluded aspira-

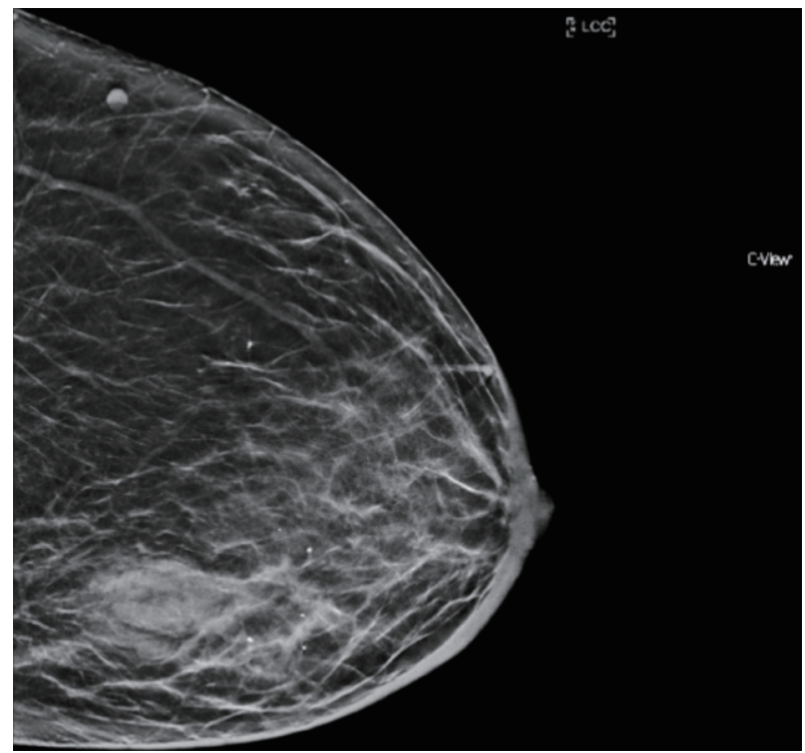

Figure 1. Mammogram of left breast (cranio-caudal view) with poorly circumscribed mass, measuring $40 \times 24 \mathrm{~mm}$, in the lateral aspect of breast at the level of the nipple. Irregular and stranded contours without suspicious calcifications were also noted. 


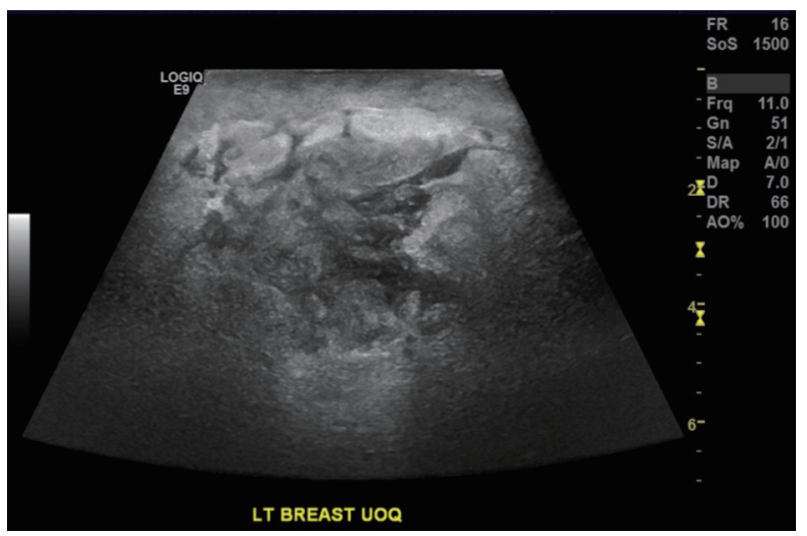

Figure 2. Ultrasound of the left breast demonstrating a lobulated, heterogeneous, hypoechoic mass lesion measuring $31 \times 17 \times 21 \mathrm{~mm}$ with accompanying distortion of the surrounding structures.

tion. The patient was advised to continue taking oral antibiotics.

The patient presented to the emergency department a few days later with ongoing discharge from her left breast. Examination revealed an open wound in the left breast with a hard, non-mobile, tender lesion palpable underneath. The wound contained central necrosis with surrounding purulent discharge and accompanying erythema extending to the nipple. Peau d'orange changes were also present (Fig. 3). The patient was noted to be hemodynamically stable. Her white cell count was elevated at $13.0 \times 10^{9} / \mathrm{L}$ and her C-reactive protein was 123 $\mathrm{mg} / \mathrm{L}$. Her blood sugar level on presentation was elevated to $25.2 \mathrm{mmol} / \mathrm{L}$.

A repeat US was conducted with no drainable collection noted. The underlying mass at the 10 o'clock position with edematous changes in the surrounding subcutaneous tissue was again noted. The patient was admitted under the general surgical team and commenced on intravenous trimethoprim and sulphamethoxazole.

A magnetic resonance imaging (MRI) was performed to aid in operative planning. The MRI revealed no evidence of malignancy or adenopathy in the right breast. The left breast contained a large collection, measuring $100 \times 40 \times 60 \mathrm{~mm}$. The MRI features were in keeping with a large abscess with accompanying feature of skin breakdown and necrosis. There was extensive inflammation and engorgement of feeding vessels with no suspicion of underlying malignancy (Fig. 4).

The patient underwent an emergency radical excision and debridement of the necrotic tissue. The necrosis extended down to the level of pectoralis fascia. Once adequately debrided, the wound was extensively irrigated with normal saline. A vacuum-assisted closure (VAC) device was placed over the wound to aid with healing. The patient was commenced on intravenous (IV) lincomycin with recurrent wound checks at VAC changes performed. There was no further necrotic tissue identified and the patient was discharged home on day 5 with planned follow-up through the general surgical outpatient clinic. Figure 5 shows wound healing 2 weeks post operation.

A tissue sample was sent to microbiology and was positive for Staphylococcus aureus and Streptococcus agalactiae. His-

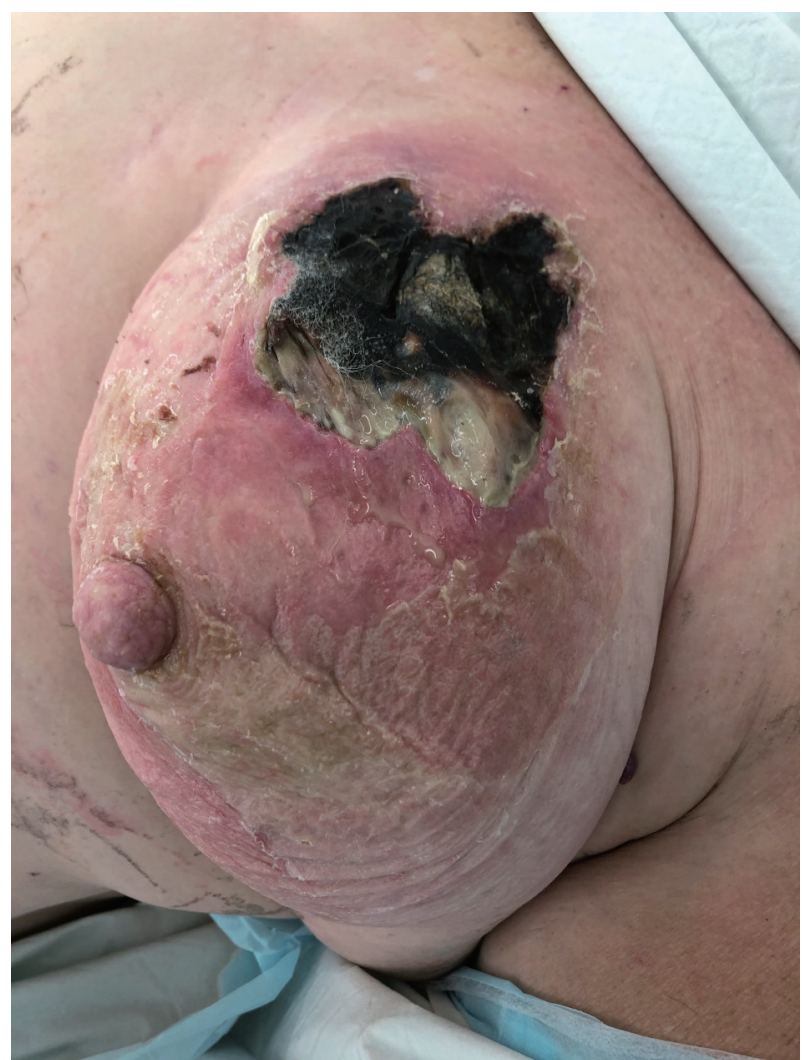

Figure 3. Clinical image of the left breast with a large wound containing central necrosis with surrounding purulent discharge and accompanying erythema extending to the nipple.

tological examination revealed the presence of an abscess with acute suppurative inflammation and ulceration of the overlying skin. Accompanying fat necrosis was also noted. There was, however, no evidence of dysplasia or malignancy.

A follow-up mammogram was conducted 6 months post debridement and the appearance was in keeping with nearly complete resolution of the previously visualized opacity (Fig. $6)$.

\section{Discussion}

Necrotizing fasciitis was first described by Hippocrates in the fifth century and later defined by Wilson in 1952 as an infective process resulting in necrosis of the fascia and subcutaneous tissue with sparing of the muscle [1-3]. It is a rapidly progressing, potentially fatal bacterial infection that spreads along fascial planes [4]. The production of bacterial toxins leads to widespread necrosis of tissue adjacent to the fascial planes and can subsequently lead to systemic toxicity and sepsis $[2,5]$. Risk factors for necrotizing fasciitis include: an immunocompromised state, underlying malignancy, diabetes mellitus, peripheral vascular disease, chronic renal failure and intravenous drug use $[5,6]$. Age is not a predictive factor in the development of necrotizing fasciitis [6].

Necrotizing fasciitis can be classified into four main cat- 


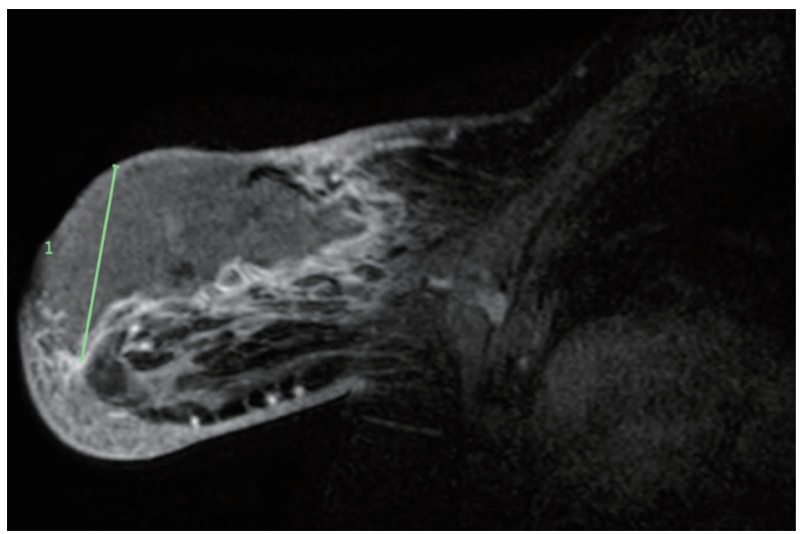

Figure 4. MRI of the left breast with a large collection, measuring100 $\times 40 \times 60 \mathrm{~mm}$. MRI features were in keeping with a large abscess with accompanying feature of skin breakdown and necrosis. MRI: magnetic resonance imaging.

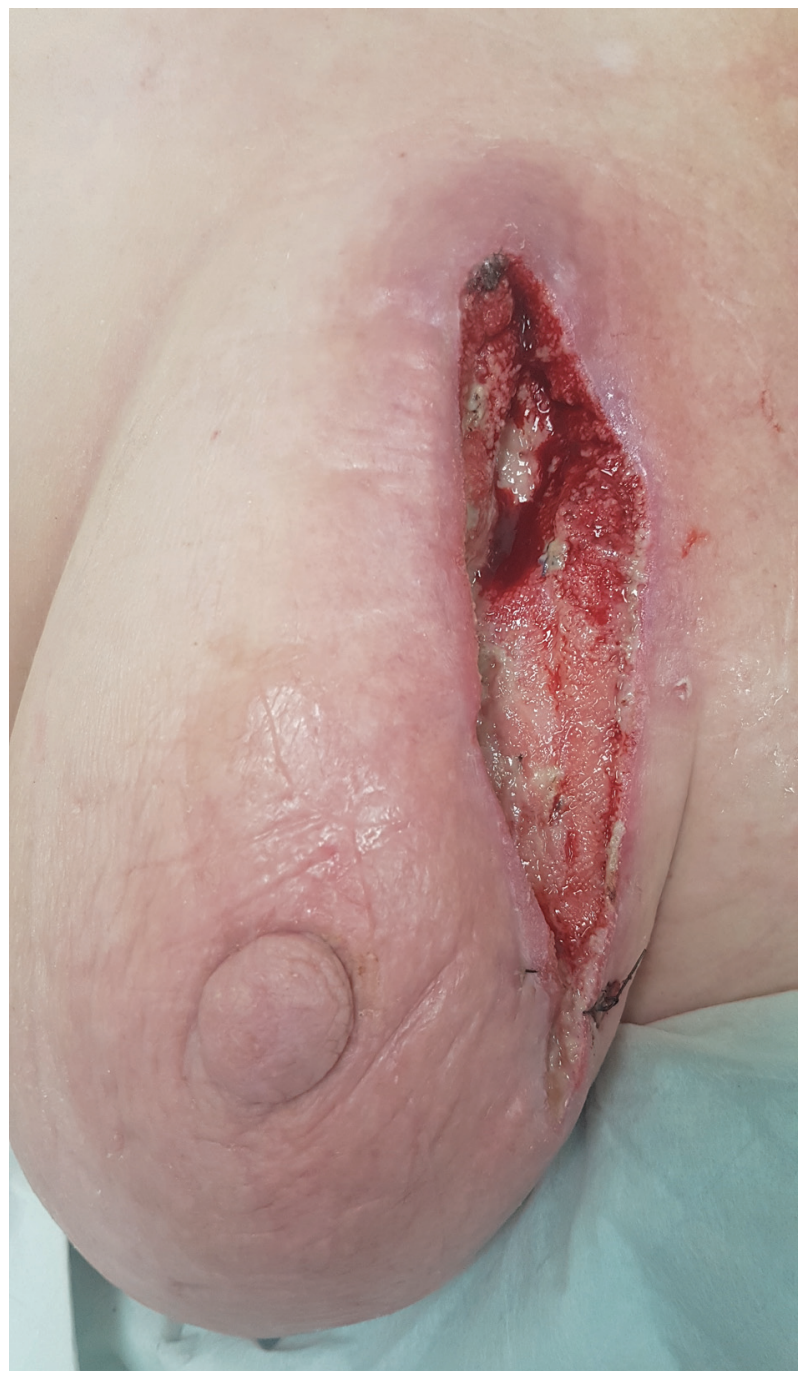

Figure 5. Clinical image of left breast 2 weeks post debridement, with resolution of the infection and healthy granulation tissue in the base of the wound.

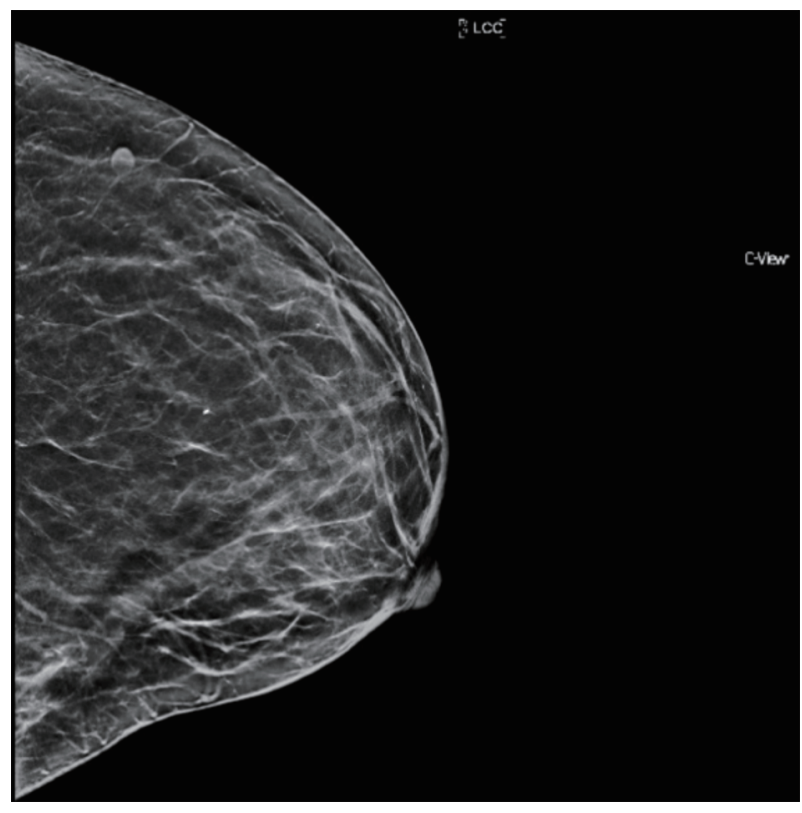

Figure 6. Follow-up mammogram (cranio-caudal view) 6 months post infection demonstrating almost complete resolution of previously visualized lesion.

egories based on etiology: types I, II, III and IV [7]. Type I necrotizing fasciitis is most commonly seen and tends to be polymicrobial in nature [7]. Indeed, it can be caused by a wide variety of bacterial agents including Gram-positive cocci such as Staphylococcus and Streptococcus species, Gram-negative bacilli such as Klebsiella species and Escherichia coli and anaerobes $[8,9]$. Type I necrotizing fasciitis typically occurs in the perineum and abdomen with the etiological bacterial agents introduced through either blunt or penetrating trauma or surgical incisions $[5,7]$.

Type II necrotizing fasciitis is typically monomicrobial in nature and is caused by group A, $\beta$-hemolytic streptococcal species such as Streptococcus pyogenes [5]. In some instances it can be associated with concurrent staphylococcal infection [6]. In contrast to type I necrotizing fasciitis, type II most commonly affects the extremities in healthy, immunocompetent hosts, and is associated with minor injuries or breaks in the skin $[5,9]$.

Type III necrotizing fasciitis is uncommon and is caused by monomicrobial infection with Gram-negative bacteria, such as Clostridium, Klebsiella or Vibrio species [1,7]. Vibrio species are commonly transmitted to humans by fish or other marine hosts [7]. Some sources also include type IV necrotizing fasciitis which is caused by fungal infections and is common following burns or large traumatic wounds $[1,10]$.

The diagnosis of necrotizing fasciitis can be challenging and a high index of suspicion is required in order to initiate prompt surgical management and decrease morbidity and mortality [10]. Typical clinical symptoms and signs include: swelling, erythema, pain out of proportion to clinical signs, and development of fluid filled bullae [7, 10]. It may be difficult to distinguish necrotizing fasciitis from cellulitis. The key pathological findings include: full thickness skin necrosis; 
perivasculitis and vasculitis, often with accompanying fibrinoid necrosis and thrombus formation; and the presence of bacterial or fungal elements on ancillary stains [7].

Necrotizing fasciitis of the breast poses a diagnostic challenge for many reasons. Firstly, the variable thickness of the underlying breast tissue between the skin and the fascia results in a delayed cutaneous reaction and widespread infection prior to diagnosis and surgical management $[7,8,11]$. As such, it can be misdiagnosed as cellulitis, mastitis, breast abscess or even inflammatory breast cancer [7, 12]. Indeed, in our case the patient was initially investigated for potential breast cancer. Following the development of infection, she was treated for cellulitis and then a breast abscess with antibiotic therapy.

Another protective factor for necrotizing fasciitis of the breast is the dense blood supply network. It includes branches from the lateral thoracic, internal thoracic, thoracoacromial branches, lateral mammary branches and internal mammary arteries [13]. This robust blood supply can delay cutaneous involvement and hence presentation due to the angiothrombotic element of the necrotizing fasciitis $[7,14]$. The effect of this delayed presentation is evident throughout the literature with the majority of patients presenting days to weeks after the initial commencement of symptoms.

The diagnosis of necrotizing fasciitis is made by clinical and surgical findings. Mostly commonly necrotizing fasciitis of the breast is unilateral [1]. Patients may have preceding symptoms such as mastitis or breast abscess [7]. Cutaneous manifestations include erythema, induration, blistering and edema [15]. These changes are non-specific, complicating the diagnosis and resulting in clinicians requiring a high index of suspicion [2].

Surgical findings include extensive necrosis along fascial planes [12]. During wound exploration tissue must be assessed for viability and all areas of necrosis must be excised [9]. In some of the previous cases described in the literature closure has been delayed to allow for repeated examination and/or debridement. Patients with widespread necrosis may require repeated debridement or mastectomy. In our case we utilized a VAC dressing to allow for regular wound checks and to allow the large defect to close by secondary intention.

A broad range of imaging modalities have been utilized in the setting of necrotizing fasciitis of the breast. In some instances, patients have undergone US, MRI or computed tomography (CT) scans. In our case, the patient was initially worked up with a US and mammogram due to the suspicion of underlying malignancy. On presentation to the emergency department, an abscess was considered in the differential diagnosis and, so, a US was conducted. In order to exclude malignancy, an MRI of the breasts was then organized. Our patient did not present with signs of systemic toxicity or sepsis and so this was reasonable investigation. Given that malignancy was excluded with an MRI we were able to proceed with debridement of necrotic tissue and avoid an unnecessary mastectomy.

Necrotizing fasciitis of the breast is rarely diagnosed with relatively few cases described in the literature. Our case is unique as it is the second case from Australia that has been published. In addition, our patient had a protracted clinical course with multiple provisional diagnoses suggested. This case highlights the need for a high index of suspicion and the role that imaging plays in the diagnosis of necrotizing fasciitis. Early diagnosis can assist in circumventing the significant morbidity and mortality associated with necrotizing fasciitis. Although not a primary concern, the cosmetic outcomes of any surgical procedures should be considered as well.

\section{Acknowledgments}

None to declare.

\section{Financial Disclosure}

None to declare.

\section{Conflict of Interest}

None to declare.

\section{Informed Consent}

It was obtained from patient prior to completing case report.

\section{Author Contributions}

All authors contributed to the conception, writing and editing of the case report. NF was involved in the acquisition, analysis and interpretation of data. All authors agreed to be accountable for all aspects of the work.

\section{References}

1. AL Shareef B, AL Saleh N. Necrotizing fasciitis of the breast: a case report and review of literature. Case Rep Surg. 2019;2019:1370680.

2. Yaji P, Bhat B, E H. Primary necrotising fasciitis of the breast: case report and brief review of literature. J Clin Diagn Res. 2014;8(7):ND01-02.

3. Wilson B. Necrotizing fasciitis. Am Surg. 1952;18(4):416431.

4. Marongiu F, Buggi F, Mingozzi M, Curcio A, Folli S. A rare case of primary necrotising fasciitis of the breast: combined use of hyperbaric oxygen and negative pressure wound therapy to conserve the breast. Review of literature. Int Wound J. 2017;14(2):349-354.

5. Ablett DJ, Bakker-Dyos J, Rainey JB. Primary necrotizing fasciitis of the breast: a case report and review of the literature. Scott Med J. 2012;57(1):60.

6. Soliman MO, Ayyash EH, Aldahham A, Asfar S. Necrotizing fasciitis of the breast: a case managed without mastectomy. Med Princ Pract. 2011;20(6):567-569.

7. Ward ND, Harris JW, Sloan DA. Necrotizing fasciitis of the breast requiring emergent radical mastectomy. 
Breast J. 2017;23(1):95-99.

8. Kaczynski J, Dillon M, Hilton J. Breast necrotising fasciitis managed by partial mastectomy. BMJ Case Rep. 2012;2012.

9. Flandrin A, Rouleau C, Azar CC, Dubon O, Giacalone PL. First report of a necrotising fasciitis of the breast following a core needle biopsy. Breast J. 2009;15(2):199201.

10. Machado NO. Necrotizing fasciitis: The importance of early diagnosis, prompt surgical debridement and adjuvant therapy. North Am J Med Sci. 2011;3(3):107.

11. Wong $\mathrm{CH}$, Tan BK. Necrotizing fasciitis of the breast. Plast Reconstr Surg. 2008;122(5):151e-152e.
12. Rajakannu M, Kate V, Ananthakrishnan N. Necrotizing infection of the breast mimicking carcinoma. Breast J. 2006;12(3):266-267.

13. Shiffman MA. Arterial blood supply of the breast. Aesthetic Surgery of the Breast: Springer; 2015. p. 43-44.

14. Yang B, Connolly S, Ball W. Necrotising fasciitis of the breast: a rare primary case with conservation of the nipple and literature review. JPRAS Open. 2015;6:15-9.

15. Aloisio da Costa Vieira R, Zucca Mathes AG, Michelli RA, Ribeiro GH, Haikel RL, Viana CR, Castro Pde T, et al. Necrotizing soft tissue infection of the breast: case report and literature review. Surg Infect (Larchmt). 2012;13(4):270-275. 